Accardi, L., Barhoumi, A. \& Ouerdiane, H., A quantum approach to Laplace operators

Accardi, L., Boukas, A. \& Franz, U., Renormalized powers of quantum white noise

Accardi, L. \& Boukas, A., Renormalized higher powers of white noise (RHPWN) and conformal field theory

Accardi, L., Fagnola, F. \& Hachicha, S., Generic qMarkov semigroups and speed of convergence of $q$-algorithms

Accardi, L., Matsuoka, T. \& Ohya, M., Entangled Markov chains are indeed entangled

Albeverio, S., Belopolskaya, Ya. \& Feller, M., Lévy-Dirichlet forms

Albeverio, S., Liang, S. \& Zegarlinski, B., Remark on the integration by parts formula for the $\phi_{3}^{4}$-quantum field model

Arveson, W., On the existence of $E_{0}$-semigroups

Banerjee, S., see Kupsch

Barhoumi, A., see Accardi

Belopolskaya, Ya., see Albeverio

Beznea, L. \& Boboc, N., Weak duality and the dual process for a semi-Dirichlet form

Boboc, N., see Beznea

Boukas, A., see Accardi

Boukas, A., see Accardi

Confortola, F., Dissipative backward stochastic differential equations in infinite dimensions

Es-Sarhir, A., Sobolev regularity of invariant measures for generalized Ornstein-Uhlenbeck operators

Fagnola, F., see Accardi
(2006) 595

9 (2006) 353

9 (2006) 567

9(2006) 379

9 (2006) 435

9(2006) 149

9 (2006) 315

9 (2006) 413

9 (2006) 435

9 (2006) 27

9 (2006) 27

9 (2006) 129

9 (2006) 353

9 (2006) 155

9 (2006) 567
9 (2006) 215

\title{
AUTHOR INDEX (Vol. 9)
}

Fan, Z., Self-similarity of free stochastic processes

Feller, M., see Albeverio

Franz, U., see Accardi

Hachaichi, R., see Jenane

Hachicha, S., see Accardi

Hiai, F. \& Ueda, Y., Free transportation cost inequalities for noncommutative multivariables

9 (2006) 391

Inahama, Y., Quasi-sure existence of Brownian rough paths and a construction of Brownian pants

Jenane, R., Hachaichi, R. \& Streit, L., Renormalisation du temps local des points triples du mouvement Brownien

Jenčová, A. \& Petz, D., Sufficiency in quantum statistical inference. A survey with examples

9 (2006) 451

9 (2006) 435

9 (2006) 129

9(2006) 547

9 (2006) 567

9 (2006) 513

Ji, U. C. \& Sinha, K. B., Uniqueness of integrands in quantum stochastic integral

Kawabi, H., A simple proof of $\log$ Sobolev inequalities on a path space with Gibbs measures

Kondratiev, Y. \& Skorokhod, A., On contact processes in continuum

Konno, N., Continuous-time quantum walks on trees in quantum probability theory

Kozyrev, S. V., see Yu. Khrennikov

Kubo, I., Kuo, H.-H. \& Namli, S., Interpolation of Chebyshev polynomials and interacting Fock spaces

Kuo, H.-H., see Kubo

Kupsch, J. \& Banerjee, S., Ultracoherence and canonical transformations
9 (2006) 331

9 (2006) 607

$9(2006) 321$

9 (2006) 187

9 (2006) 199

9(2006) 361

9 (2006) 361

9(2006) 413
9 (2006) 287 
Lenczewski, R. \& Sałapata, R., Discrete interpolation between monotone probability and free probability

Liang, S., see Albeverio

Márquez-Carreras, D., On the asymptotics of the density in perturbed SPDE's with spatially correlated noise

Matsui, T., BEC of free bosons on networks

Matsuoka, T., see Accardi

Muhly, P. S., Skeide, M. \& Solel, B., Representations of $\mathcal{B}^{a}(E)$

Namli, S., see Kubo

Obata, N., A note on Konno's paper on quantum walk

Obrezkov, O. O., Non-selfadjoint extensions of the LévyLaplacian and the LévyLaplace equation

Ohya, M., see Accardi

Øksendal, B., Proske, F. \& Signahl, M., The Cauchy problem for the wave equation with Lévy noise initial data

Ouerdiane, H., see Accardi

Petz, D., see Jenčová

Prokhorenko, D. V., Squares of white noise, $\operatorname{SL}(2, \mathbb{C})$ and Kubo-Martin-Schwinger states

Proske, F., see Øksendal

Rozikov, U. A. \& Suhov, Y. M., Gibbs measures for SOS mod-

els on a Cayley tree

9 (2006) 471

Sałapata, R., see Lenczewski

Signahl, M., see Øksendal

9 (2006) 77

9(2006) 149

9(2006) 271

9 (2006) 1

9 (2006) 379

9 (2006) 47

9(2006) 361

9 (2006) 299

9 (2006) 67

9(2006) 379

9 (2006) 249

9(2006) 215

9 (2006) 331

9 (2006) 491

9 (2006) 249
Sinha, K. B., see Ji

Skeide, M., see Muhly

Skeide, M., A simple proof of the fundamental theorem about Arveson systems

Skeide, M., A simple proof of the fundamental theorem about Arveson systems (Errata)

Skeide, M., Existence of $E_{0^{-}}$ semigroups for Arveson systems: Making two proofs into one

Skeide, M., The index of (white) noises and their product systems

Skorokhod, A., see Kondratiev

Śniady, P., Gaussian fluctuations of representations of wreath products

Solel, B., see Muhly

Stan, A. I., Best constants in norms of Wick products

Streit, L., see Jenane

Suhov, Y. M., see Rozikov

Ueda, Y., see Hiai

Wang, F.-Y., The stochastic order and critical phenomena for superprocesses

Yu. Khrennikov, A. \& Kozyrev, S. V., Ultrametric random field Zegarlinski, B., see Albeverio
9 (2006) 77

9 (2006) 249

9 (2006) 607

9 (2006) 47

9 (2006) 305

9 (2006) 489

9 (2006) 373

9(2006) 617

9(2006) 187

9 (2006) 529

9 (2006) 47

9 (2006) 169

9 (2006) 547

9 (2006) 471

9 (2006) 391

9 (2006) 107

9(2006) 199

9(2006) 149 